\title{
Sobrevida y factores pronósticos de 13 pacientes con carcinoma espinocelular oral diagnosticados en el CRS Cordillera Oriente, entre los años 2001 - 2015
}

\author{
Survival and prognostic factors in 13 patients with oral squamous cell carcinoma \\ diagnosed at the CRS Cordillera Oriente, between the years 2001 - 2015
}

Bárbara Feldman F$F^{1}$, Alejandra Castro A², Olga Salinas $\mathrm{F}^{2}$, Daniel Sotomayor $\mathrm{J}^{3}$.

\begin{abstract}
RESUMEN
Introducción: El carcinoma espinocelular (CEC) es una neoplasia maligna derivada del epitelio plano estratificado. Hoy en día ha adquirido mayor importancia debido a que es el cáncer oral más frecuente y su sobrevida sobre los 5 años se ha mantenido baja.

objetivo: Generar información que oriente a odontólogos y médicos en la detección y manejo oportuno del CEC oral analizando la sobrevida según características demográficas y clínicas de pacientes con esta enfermedad atendidos en el CRS Cordillera Oriente.

Materiales y métodos: Estudio descriptivo, retrospectivo de tipo serie de casos. La muestra correspondió al total de pacientes diagnosticados con CEC oral en el CRS Cordillera Oriente entre enero de 2001 y mayo de 2015. Para el estudio epidemiológico de la muestra se utilizó estadística descriptiva y para el análisis de la sobrevida se utilizó el método de Kaplan Meier.

Resultados: La mayor parte de la muestra fueron hombres (8/13) y mayores de 60 años (7/13). La edad promedio fue de 63,6 años. La presentación más común fue la úlcera crónica (6/13) y la localización más frecuente, el labio (4/13). En su mayoría fueron tumores bien diferenciados (9/13), un estado del tumor primario en etapa pT1 o pT2 (7/8) y ausencia de metástasis en nodos regionales (6/9). Se encontró una distribución homogénea respecto a la etapa TNM. La sobrevida general a los 5 años fue de $57,9 \%$. La sobrevida más baja a los 5 años se presentó en pacientes de sexo femenino, menores de 45 años, fumadores, con presentación de masa exofítica, con tumores de menor grado de diferenciación histológica, con un estado del tumor primario avanzado, con presencia de metástasis en nodos regionales y que se encontraban en una etapa TNM avanzada.

Conclusiones: La prevalencia de CEC oral en este centro es baja, sin embargo, es de relevancia dado la reducida sobrevida sobre los 5 años. Esto pone de manifiesto la necesidad de un diagnóstico y tratamiento oportunos. La información presentada es de utilidad para la identificación de la población en riesgo de CEC oral, pudiendo ayudar en el desarrollo de programas de tamizaje. El limitado número de casos puede explicar las diferencias observadas respecto a la literatura nacional e internacional lo que genera la necesidad de realizar nuevos estudios.
\end{abstract}

Palabras clave: Sobrevida, carcinoma espinocelular, cáncer oral, pronóstico.

\footnotetext{
Interna Odontología Universidad del Desarrollo.

2 Cirujana-Dentista Servicio Odontología CRS Cordillera Oriente de Peñalolén.

3 Interno Medicina Universidad del Desarrollo.
} 


\section{ABSTRACT}

Introduction: Squamous cell carcinoma (SCC) is a malignant neoplasm derived from stratified squamous epithelium. Today it has become more important because it is the most common oral cancer and its survival over five years has remained low.

Aim: Produce information to guide dentist and physicians in the prompt detection and management of oral SCC through the analysis of survival according to demographic and clinical characteristics of patients diagnosed at the CRS Cordillera Oriente.

Methods: We perform a descriptive, retrospective study of case series. Patients diagnosed with oral SCC at the CRS Cordillera Oriente, between January 2001 and May 2015 were selected. We used descriptive statistics for the epidemiological study sample and the Kaplan Meier method for the survival analysis.

Results: The study population was mainly men gender (8/13) and over 60 years (7/13). The average age was 63,6 years. The most common presentation was chronic ulcer (6/13) and the most common site, the lip (4/13). Most of the sample had a well differentiated tumor (9/13), a primary tumor stage pT1 or pT2 (7/8) and absence of metastases in regional nodes (6/9). A homogeneous distribution with respect to the TNM stage was found. The overall survival at 5 years was $57,9 \%$, the lowest rates were in the female gender, group under 45 years, smokers, exophytic mass, poorly differentiated tumor, advanced primary tumor, presence of regional metastases in nodes and advanced TNM stage.

Conclusions: The prevalence of oral SCC in this center is low, however, it is relevant given the reduced survival over 5 years. This highlights the need for early diagnosis and treatment. The information presented is useful for identifying population at risk or oral SCC, and may assist in the development of screening programs. The limited number of cases may explain the observed differences regarding the national and international literature wich generates the need for further studies.

Key words: Survival, squamous cell carcinoma, mouth neoplasms, prognosis.

\section{INTRODUCCIÓN}

A nivel mundial, el cáncer de la cavidad oral y de faringe corresponde al décimo cáncer con mayor incidencia y a la séptima causa de mortalidad producida por cáncer. En Chile, se estima que la incidencia de cáncer de labio y cavidad oral es de 1,3 casos por cada 100.000 habitantes y la mortalidad de 0,6 casos por cada 100.000 habitantes ${ }^{1}$. Dentro del cáncer oral, el CEC es el más frecuente, correspondiendo al $90 \%$ de las neoplasias en dicho territorio².

Esta enfermedad afecta con mayor frecuencia a los hombres, en una razón hombres:mujeres de 1,5:13. La incidencia general de CEC es de 8,8 casos por cada 100.000 habitantes en hombres y de 5,1 casos por cada 100.000 habitantes en mujeres ${ }^{1}$. En relación a la edad, el $90 \%$ de los pacientes diagnosticados con CEC son mayores de 45 años 4 .

EI CEC es una neoplasia multifactorial, se han establecido como factores de riesgo el consumo de tabaco, el alto consumo de alcohol, la combinación de consumo de tabaco y alcohol, la presencia de lesiones 0 condiciones potencialmente malignas, la historia previa de cáncer oral 0 en el tracto aero-digestivo y la edad. Otros posibles factores reportados son las deficiencias vitamínicas, la infección por virus papiloma humano (HPV), la exposición excesiva a luz solar, la predisposición genética, la exposición a contaminación ambiental y la sepsis crónica oral ${ }^{5}$.

Esta patología se presenta en variadas formas clínicas, lo cual está relacionado con la localización del tumor, el tiempo de evolución, la presencia de lesiones premalignas y los factores de riesgo asociados. Puede observarse como una lesión leucoplásica, una 
lesión eritroplásica, una lesión leucoeritroplásica, como una úlcera de aspecto necrótico con bordes blanco-grisáceos, elevados, evertidos e indurados o como una lesión lobulada irregular y exofítica de base amplia 6 . En etapas más avanzadas, el carcinoma evoluciona a una lesión proliferativa ulcerada con áreas de necrosis que se extiende a estructuras adyacentes como hueso, músculos y piel7. Las localizaciones más frecuentes son el labio inferior, los bordes laterales de la lengua y el piso de boca ${ }^{8}$.

A pesar de los avances en técnicas quirúrgicas y terapias adyuvantes los porcentajes de sobrevida se han mantenido relativamente estables ${ }^{4}$. La literatura disponible reporta una sobrevida a los 5 años en un rango de $28,6 \%$ a $76,8 \%$, se ha observado que el diagnóstico oportuno de la enfermedad se relaciona con una mayor sobrevida ${ }^{4,9-20}$. La Tabla 1 ilustra la sobrevida a los 5 años por CEC reportada por diversos autores. Son acotados los estudios sobre la prevalencia de esta patología en Chile, más aún los estudios de sobrevida y factores pronósticos. Cabe añadir que esta población no ha sido estudiada previamente.

\section{OBJETIVO}

El objetivo de esta investigación es generar información que oriente a odontólogos y médicos en la detección y manejo oportuno del CEC oral por medio de un análisis epidemiológico y de sobrevida según características demográficas y clínicas de pacientes con esta enfermedad atendidos en el CRS Cordillera Oriente de Peñalolén.

\section{MATERIAL Y MÉTODO}

Se realizó un estudio de carácter observacional, descriptivo y retrospectivo de tipo serie de casos. La muestra estudiada correspondió al total de la población de pacientes diagnosticados con carcinoma espinocelular oral en el CRS Cordillera Oriente de Peñalolén entre enero de 2001 y mayo de 2015. Como criterio de inclusión se utilizó a pacientes diagnosticados con carcinoma espinocelular oral en etapa de carcinoma in situ o carcinoma invasivo confirmado mediante una biopsia incisional en el servicio. Se excluyeron pacientes con diagnóstico de cáncer oral diferente a carcinoma espinocelular, pacientes con carcinoma oral en una localización diferente a C00, C01, C02, C03, C04, C05 y C06 según la Clasificación Internacional de Enfermedades (CIE-10), pacientes que no tuviesen ficha clínica en el servicio o que ésta estuviese incompleta en relación a sexo, edad, presentación clínica y localización anatómica, y pacientes con tumores como metástasis de otro tumor.

Se obtuvo la aprobación del Comité de Ética Científico del Servicio de Salud Metropolitano Oriente y una resolución efectuada por el CRS Cordillera Oriente que autorizó la realización del proyecto. Se accedió a la base de datos de la unidad dental del CRS Cordillera Oriente y posteriormente, a las fichas clínicas de cirugía de cabeza y cuello en el Hospital del Salvador.

Se recolectaron datos acerca del sexo (masculino o femenino), edad (años), hábitos (tabaco,

Tabla 1. Sobrevida de pacientes con CEC a los $\mathbf{5}$ años en la literatura

\begin{tabular}{|lcccc|}
\hline Autores & País & Año & (n) & Sobrevida general a los 5 años (\%) \\
\hline Arduino P. et al. & Italia & 2008 & 334 & 76,8 \\
Jan JC. et al. & Taiwan & 2011 & 415 & 71 \\
Takahashi M. et al. & Japón & 2014 & 502 & 68,4 \\
Garzino - Demo P. et al. & Italia & 2006 & 245 & $63 \%$ \\
Grimm M. & Alemania & 2012 & 484 & 62 \\
Oliveira M. et al. & Uruguay & 2015 & 200 & 58,5 \\
Arriagada O. et al. & Chile & 2010 & 36 & 58,4 \\
Wang B. et al. & China & 2013 & 275 & 54,5 \\
Rogers S. et al. & Reino Unido & 2009 & 489 & 56 \\
Silva L. et al. & Portugal & 2014 & 128 & 50,5 \\
Sklenicka S. et al. & EE.UU. & 2010 & 155 & 48 \\
Momares B. et al. & Chile & 2014 & 161 & 46 \\
Oliveira L. et al. & Brasil & 2008 & 500 & 28,6 \\
\hline
\end{tabular}


alcohol, ambos, ninguno), presentación clínica (lesión leucoplásica, lesión eritroplásica, lesión leucoeritroplásica, úlcera crónica, masa exofítica, otros), localización anatómica (labio, lengua, paladar, encía o reborde, piso de boca, combinación piso de boca y lengua, otros), grado histológico según la clasificación internacional histológica de tumores de la OMS (bien diferenciado, moderadamente diferenciado, poco diferenciado), tamaño de la lesión (milímetros), estado del tumor primario según el sistema de clasificación TNM realizado por la AJCC (Tis, T1, T2, T3 y T4), estado de los nodos regionales (ausencia o presencia de metástasis), etapa TNM según clasificación TNM (etapa 0, etapa I, etapa II, etapa III y etapa IV).

Para conocer el estado del paciente se obtuvieron los certificados de defunción con causa de muerte a partir del Registro Civil de Chile. Los pacientes que permanecieron vivos fueron estudiados desde la fecha 31 de agosto de 2015 retrospectivamente hasta la fecha de su diagnóstico.

Se realizó un análisis exploratorio mediante estadística descriptiva para caracterizar variables demográficas y clínicas de la muestra. Para el análisis de la sobrevida general se utilizó el método de Kaplan Meier. Para la estimación de la sobrevida a un determinado tiempo se calculó un intervalo de confianza del $95 \%$.

\section{RESULTADOS}

\section{Características epidemiológicas}

Se obtuvo una muestra de 13 pacientes con carcinoma espinocelular oral. La mayoría de los casos se encontraron en hombres (8/13), en una relación hombres:mujeres de 1,6:1. La edad promedio fue de $63,6 \pm 19,72$ años, con un rango de 28 a 88 años. El promedio de edad en hombres fue de 56,1 años mientras que, en mujeres fue de 75,8 años. La Tabla 2 muestra la distribución de las características demográficas encontradas. La mayor frecuencia de casos se encontró en el intervalo de pacientes mayores de 60 años, seguido de pacientes entre 45 y 60 años, existiendo la menor cantidad de casos en menores de 45 años.

Se pudo evaluar el hábito de fumar y de beber alcohol en 12 pacientes ya que una ficha clínica no
Tabla 2. Distribución de características demográficas de la muestra

\begin{tabular}{|lcc|}
\hline Variables & $(\mathrm{n})$ & $(\%)$ \\
\hline Sexo & $\mathrm{n}: 13$ & \\
Masculino & 8 & 61,5 \\
Femenino & 5 & 38,4 \\
Edad & $\mathrm{n}: 13$ & \\
$<45$ años & 2 & 15,3 \\
$45-60$ años & 4 & 30,7 \\
$>60$ años & 7 & 53,8 \\
Hábitos & $\mathrm{n}: 12$ & \\
Tabaco & 3 & 25 \\
Alcohol & 0 & 0 \\
Tabaco y alcohol & 0 & 0 \\
No hay hábitos & 9 & 75 \\
\hline
\end{tabular}

presentaba la información. Los 3 pacientes que presentaron hábito tabáquico fueron de sexo masculino. No se encontraron pacientes que relataran el hábito de beber alcohol.

La presentación clínica más común fue la úlcera crónica. La Tabla 3 muestra la distribución de las características clínicas e histológicas de la muestra. La localización anatómica más frecuente fue en labio (4/13), seguido de la lengua (3/13). En relación al grado histológico, la mayor parte de la muestra presentó un grado bien diferenciado (9/13), seguido de un patrón moderadamente diferenciado (4/13). El tamaño promedio de las lesiones fue 30 milímetros $(\mathrm{mm})$. Las mujeres presentaron un tamaño promedio de $32 \mathrm{~mm}$ y los hombres de $28 \mathrm{~mm}$. De acuerdo a la localización anatómica, las lesiones en labio midieron en promedio $16,5 \mathrm{~mm}$ y las lesiones intraorales $34 \mathrm{~mm}$. La mayoría de los pacientes presentaron lesiones en etapa pT1 o pT2 (7/8) y nodos regionales no afectados (6/9) al momento de la cirugía. Respecto a la etapa TNM, se observa una distribución homogénea de la muestra.

\section{Sobrevida}

La sobrevida se pudo evaluar en el total de la muestra. Utilizando la fecha 31 de agosto de 2015 como fecha de cierre, se logró un seguimiento de 5.053 días, una mediana de 887 días y un tiempo mínimo de 116 días. Se logró estimar que el 75\% de la muestra se mantuvo viva a los 629 días $(1,7$ 
Tabla 3. Distribución de características clínicas e histológicas de la muestra

\begin{tabular}{|c|c|c|c|c|}
\hline Variable & Hombres (n) & Mujeres (n) & Total (n) & Total $(\%)$ \\
\hline \multicolumn{5}{|l|}{ Presentación clínica (n: 13) } \\
\hline Lesión leucoplásica & 1 & 0 & 1 & 7,6 \\
\hline Lesión eritroplásica & 0 & 0 & 0 & 0 \\
\hline Lesión leuco-eritroplásica & 0 & 4 & 4 & 30,7 \\
\hline Úlcera crónica & 5 & 1 & 6 & 46,1 \\
\hline Masa exofítica & 2 & 0 & 2 & 15,3 \\
\hline \multicolumn{5}{|l|}{ Localización anatómica (n: 13) } \\
\hline Labio & 4 & 0 & 4 & 30,7 \\
\hline Lengua & 2 & 1 & 3 & 23 \\
\hline Paladar & 1 & 0 & 1 & 7,6 \\
\hline Encía & 0 & 2 & 2 & 15,3 \\
\hline Piso de boca & 0 & 2 & 2 & 15,3 \\
\hline Piso de boca y lengua & 1 & 0 & 1 & 7,6 \\
\hline \multicolumn{5}{|l|}{ Grado histológico (n: 13) } \\
\hline Bien diferenciado & 5 & 4 & 9 & 69,2 \\
\hline Moderadamente diferenciado & 3 & 1 & 4 & 30,7 \\
\hline Poco diferenciado & 0 & 0 & 0 & 0 \\
\hline \multicolumn{5}{|l|}{ Estado tumor primario (n: 8) } \\
\hline Tis & 0 & 1 & 1 & 12,5 \\
\hline $\mathrm{T} 1$ & 2 & 0 & 2 & 25 \\
\hline T2 & 1 & 3 & 4 & 50 \\
\hline T3 & 1 & 0 & 1 & 12,5 \\
\hline T4 & 0 & 0 & 0 & 0 \\
\hline \multicolumn{5}{|l|}{ Estado nodos regionales (n: 9 ) } \\
\hline NO & 3 & 3 & 6 & 66,6 \\
\hline $\mathrm{N}_{+}$ & 2 & 1 & 3 & 33,3 \\
\hline \multicolumn{5}{|l|}{ Clasificación TNM (n: 9) } \\
\hline Etapa 0 & 0 & 1 & 1 & 11,1 \\
\hline Etapa 1 & 2 & 0 & 2 & 22,2 \\
\hline Etapa 2 & 0 & 2 & 2 & 22,2 \\
\hline Etapa 3 & 2 & 0 & 2 & 22,2 \\
\hline Etapa 4 & 1 & 1 & 2 & 22,2 \\
\hline
\end{tabular}

NO: ausencia de metástasis en nodos regionales; N+: presencia de metástasis en nodos regionales.

años). El tiempo medio de sobrevida fue de 1.566 días (4,2 años). Al realizar el análisis de Kaplan Meier se obtuvo una sobrevida a los 5 años de $57,9 \%$. La Figura 1 muestra la curva de sobrevida general.

En relación al sexo, se encontró que el tiempo promedio de sobrevida en mujeres fue de 3,4 años y en hombres de 4,7 años. La sobrevida a los 5 años en mujeres fue de $40 \%$ y en hombres de $72,2 \%$ (Figura 2).

Según grupo etario, a los 3 años en pacientes menores de 45 años la sobrevida fue de $50 \%$, a 3 y 5 años en pacientes entre 45 y 60 años fue de $66,7 \%$ y en mayores de 60 fue de $57,1 \%$ (Figura 3 ).

Al analizar el hábito tabáquico, se obtuvo un tiempo promedio de sobrevida de 4,3 años en pacientes no fumadores y de 1,8 años en pacientes fumadores. La sobrevida a los 4 años en pacientes fumadores fue de $50 \%$ y en no fumadores fue de $54,5 \%$.

La Figura 4 ilustra la sobrevida según la presentación clínica. La sobrevida al año y a los 5 años de pacientes con úlcera crónica fue de 66,7\%, en aquellos con masa exofítica fue de $50 \%$ y en pacientes con lesión leucoeritroplásica fue de $75 \%$ y $50 \%$, respectivamente. El paciente con lesión leucoplásica fue diagnosticado en el año 2015 y permanece vivo.

Respecto a la localización anatómica, la sobrevida a los 5 años de pacientes con lesiones en lengua, labio y reborde fue de $66,7 \%, 100 \%$ y $50 \%$, respectivamente. El fallecimiento de pacientes con localización en piso de boca sucedió antes de los 5 años. La sobrevida al año para lesiones en piso 
de boca fue de $50 \%$. La localización en paladar se observó en un paciente el cual falleció a los 9 meses del diagnóstico. Por su parte, la localización en piso de boca y lengua se encontró en un paciente diagnosticado en 2015 y sigue vivo.

En cuanto al grado de diferenciación histológico, la sobrevida a los 3 y 5 años de pacientes con carcinoma bien diferenciado fue de $70,1 \%$ y en carcinomas con diferenciación moderada la sobrevida a los 3 años fue de $25 \%$, lo que se aprecia en la Figura 5.

La sobrevida a los 5 años de los pacientes con lesiones en $\mathrm{T} 1$ fue del $100 \%$, en pacientes con lesiones en T2 del $50 \%$ y el paciente en T3 falleció antes de los 5 años (Figura 6).

El tiempo promedio de sobrevida en pacientes con NO fue de 6,3 años y en pacientes con metástasis en nodos regionales fue de 3 años. La sobrevida a los 3 y 5 años de pacientes sin metástasis en nodos regionales fue de $66,7 \%$ y en pacientes con metástasis la sobrevida a los 3 años fue de $33,3 \%$ (Figura 7 ).

La Figura 8 muestra la curva de sobrevida según la etapa TNM. El tiempo promedio de sobrevida en pacientes en etapa I y II fue de 7,5 años y en pacientes en etapa III y IV de 1,6 años. La sobrevida a los 3 y 5 años en pacientes en etapa I fue del $100 \%$, en pacientes en etapa II ambas fueron del $50 \%$. La sobrevida a los 3 años de pacientes en etapa III fue de $50 \%$. Todos los casos en etapa IV fallecieron antes de los 2 años.

Finalmente, en los 5.053 días de seguimiento, se mantuvo vivo el $61,6 \%$ de la muestra, en tanto, el $38,4 \%$ falleció. De estos últimos, $23 \%$ murió debido al carcinoma espinocelular y $15,3 \%$ debido a otras causas.

\section{DISCUSIÓN}

En la presente serie de casos se logró estimar que el $75 \%$ de la muestra se mantuvo viva a los 1,7 años. Resultado similar fue reportado por Momares B. y cols ${ }^{4}$, quienes encontraron que el $75 \%$ de su muestra se mantuvo viva al año. La sobrevida general concuerda con estudios previos que describen una sobrevida similar a los 5 años ${ }^{10,14-16,19}$. Mientras que la cifra obtenida es menor a lo reportado por algunas investigaciones ${ }^{9,11-13,18}$. Otros autores

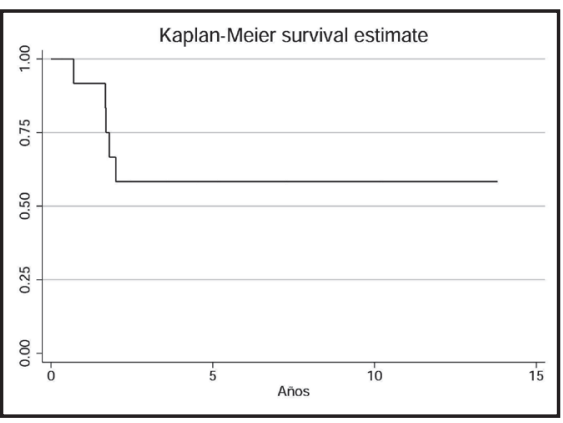

Figura 1. Curva de sobrevida general de Kaplan Meier. Leyenda: $(n=13)$.

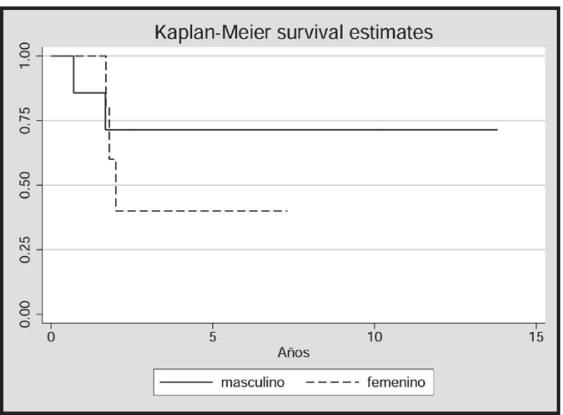

Figura 2. Curva de sobrevida de Kaplan Meier según sexo. Leyenda: (masculino, $n=8$; femenino, $n=5$ ).

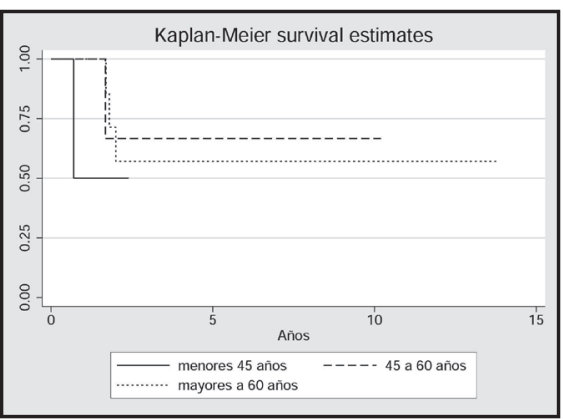

Figura 3. Curva de sobrevida de Kaplan Meier según grupo etario. Leyenda: (menores de 45 años, $n=2 ; 45$ a 60 años, $n$ $=4$; mayores de 60 años, $n=7$ ).

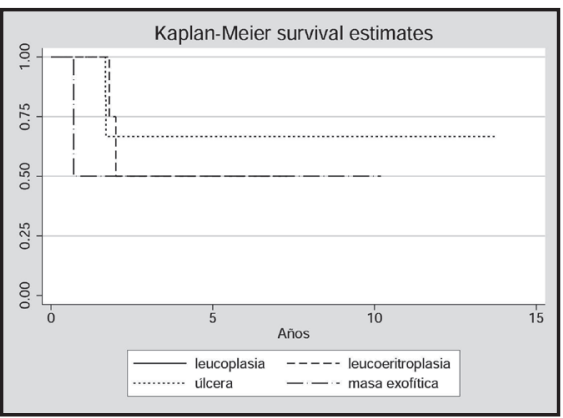

Figura 4. Curva de sobrevida de Kaplan Meier según presentación clínica. Leyenda: (leucoplasia, $n=1$; leucoeritroplasia, $n=4$; úlcera, $\mathrm{n}=6$; masa exofítica, $\mathrm{n}=2$ ). 


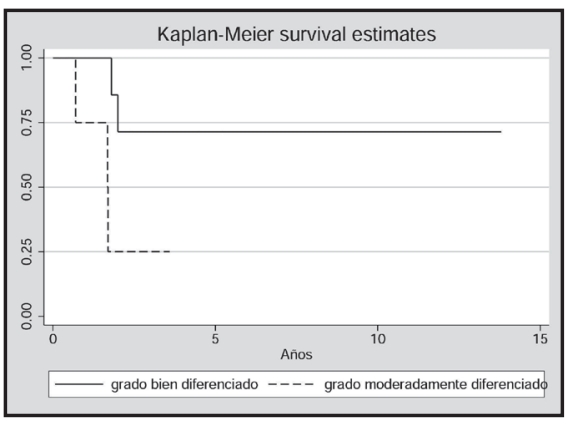

Figura 5. Curva de sobrevida de Kaplan Meier según grado histológico. Leyenda: (bien diferenciado, $n=9$; moderadamente diferenciado, $n=4$ ).

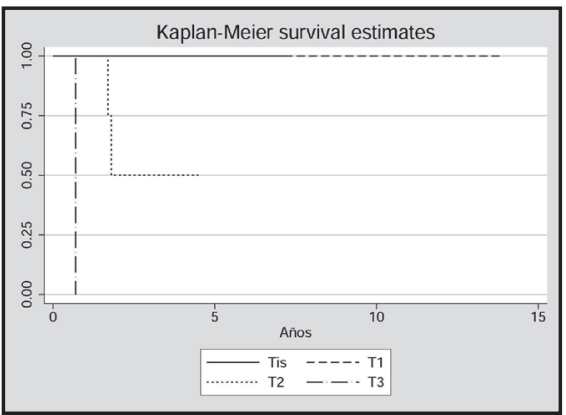

Figura 6. Curva de sobrevida de Kaplan Meier según tumo primario. Leyenda: (Tis, $n=1 ; T 1, n=2 ; T 2, n=4 ; T 3, n=1$ ).

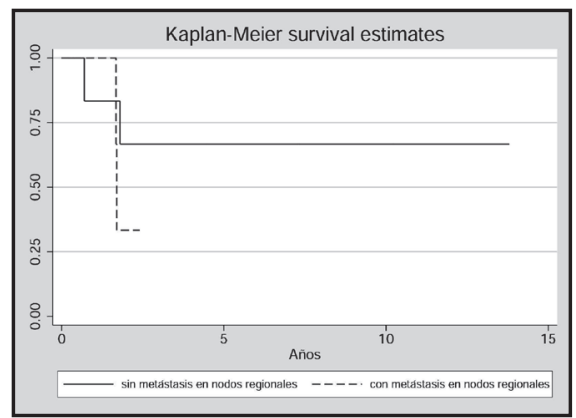

Figura 7. Curva de sobrevida de Kaplan Meier según estado de nodos regionales. Leyenda: (sin metástasis en nodos regionales, $n=6$; con metástasis en nodos regionales, $n=3$ ).

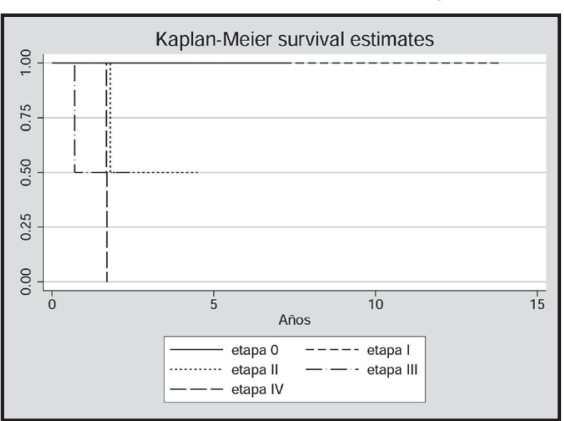

Figura 8. Curva de sobrevida de Kaplan Meier según etapa TNM. Leyenda: (etapa $0, n=1$; etapa I, $n=2$; etapa II, $n=2$; etapa III, $\mathrm{n}=2$; etapa IV, $\mathrm{n}=2$ ). encontraron sobrevidas más bajas en comparación al presente trabajo $0^{4,17,20}$. Esta inconsistencia puede deberse a varios factores, entre ellos, el acceso al tratamiento en los diversos países, la pesquisa en estadios más tempranos en países más desarrollados, el carácter del centro de salud donde se recogieron los datos puesto que, centros de referencia oncológica podrían exhibir una menor sobrevida debido a la complejidad de los casos y por diferencias genéticas y medio ambientales en las poblaciones estudiadas. Cabe señalar que la muestra fue estudiada en un establecimiento que no es centro de referencia oncológico, lo que podría explicar la mayor sobrevida respecto a otros estudios chilenos. La mortalidad específica por CEC obtenida fue de $23 \%$, lo cual coincide con algunas publicaciones 9,11-13,16. $^{2}$.

El análisis según sexo reveló una tendencia de las mujeres a presentar una menor sobrevida. Esto concuerda con lo descrito por Massano J. y cols ${ }^{21}$ en su revisión de la literatura. Contrariamente a lo observado en esta investigación, Momares B. y cols ${ }^{4}$ y Vallecillo M. y cols ${ }^{22}$ reportaron que los hombres tendían a tener la menor sobrevida. Los resultados del presente estudio podrían deberse a que las mujeres presentan un retraso en el diagnóstico mayor a los hombres ${ }^{23}$. La mayoría de los estudios coincide en que el sexo no está asociado a la sobrevida $a^{4,9-13,24}$.

La asociación entre la edad y el pronóstico parece ser controversial en la literatura. Este estudio observó que las personas menores de 45 años tendían a fallecer antes y presentar una menor sobrevida, lo cual difiere con lo encontrado en trabajos previos. Momares B. y cols ${ }^{4}$ concluyeron que las personas menores de 45 años exhibían la mayor sobrevida. Por su parte, otros estudios afirman que la edad mayor a 70 años supone el peor pronósti$\mathrm{CO}^{11,19,20}$. Los resultados podrían tener origen en las características de la muestra lo que tendría directa relación con la amplia desviación estándar de edad, mayor que la observada en estudios previos, 0 a que ambos casos de pacientes menores de 45 años se presentaron en sitios anatómicos de peor pronóstico. Múltiples estudios han demostrado que no existe asociación significativa entre la edad y la sobrevida $4,9,10,12-14$.

Los pacientes fumadores presentaron una menor sobrevida que los pacientes no fumadores, lo 
cual también fue descrito por Momares y cols ${ }^{4}$. Los datos observados en la presente investigación deben ser interpretados con prudencia, puesto que no se pudo medir adecuadamente la exposición al hábito debido a su carácter retrospectivo. Diversos estudios reportan que el hábito de fumar no estaría asociado a la sobrevida ${ }^{4,10,11,22}$.

Escasos estudios describen la frecuencia de casos según la presentación clínica, aun menos trabajos analizan su relación con la sobrevida. La curva de Kaplan Meier según la presentación clínica, muestra una tendencia de los pacientes con masa exofítica a fallecer antes y presentar una menor sobrevida. Este resultado no coincide con los encontrados por Oliveira M. y cols ${ }^{15}$ quienes afirman que la presentación como úlcera supone la menor sobrevida. Lo observado en este estudio puede deberse al número limitado de casos como también a que es una presentación clínica que se da en estadios más avanzados de la enfermedad.

Respecto a la localización anatómica, los resultados ilustran una tendencia de la localización en labio a presentar la mayor sobrevida en la muestra. Este hallazgo es consistente con los encontrados por otras investigaciones ${ }^{4,14,20,25}$. Una explicación tentativa a lo encontrado es que las lesiones en labio son más visibles por lo que los pacientes pueden consultar en estadios más tempranos de la enfermedad. La menor sobrevida encontrada para lesiones en piso de boca había sido descrita previamente por otros autores ${ }^{4,20,25}$. Lo encontrado podría explicarse debido al mayor drenaje linfático del sitio anatómico lo que incrementa su posibilidad de metástasis. El hallazgo para la localización en el paladar debe ser interpretado con precaución dado que se presentó en un paciente de la muestra, el cual fue diagnosticado en una etapa TNM III. Este resultado se contrapone a lo descrito por otros estudios que afirmaron que la menor sobrevida se observaba en lesiones de piso de boca 0 lengua $a^{4,20,25}$ y en lesiones en encía o reborde alveo$\operatorname{lar}^{12,22}$. Esta inconsistencia con la literatura puede deberse al número limitado de casos de la muestra. Varios estudios revelan que existe una asociación significativa entre la localización anatómica y la sobrevida ${ }^{10,12,14,18,20,22,24,26}$.

Numerosos estudios han afirmado que existe una asociación entre el grado histológico y la sobrevida, de tal forma que un menor grado de dife- renciación supone una menor sobrevida9-12,17-19,24,27, esto se corresponde con lo observado en la presente serie de casos. De forma similar, múltiples publicaciones coinciden en que un estado avanzado del tumor primario estaría asociado a una menor sobrevida ${ }^{9-13,15,19,22}$, lo cual fue observado en esta muestra. La metástasis en nodos regionales ha sido ampliamente aceptada como un factor pronóstico en la sobrevida. La curva según el estado de nodos regionales ilustró una tendencia de los pacientes sin metástasis a presentar una mayor sobrevida. Esto se corresponde con lo descrito por diversos autores ${ }^{9-13,15,17-19,22}$. Según etapa TNM, se exhibe una tendencia de los pacientes en etapas más avanzadas (III y IV) a presentar una menor sobrevida. Lo observado es consistente con lo descrito en la literatura $10,12,13,15,17,18,24$.

En relación al perfil epidemiológico de la muestra, se encontró que la mayoría de los casos se presentaban en hombres (8/13) lo cual coincide con otros trabajos ${ }^{4,9-12,14,28-35}$. La relación hombres:mujeres en el presente estudio fue 1,6:1, similar a lo descrito por Feller L. y cols ${ }^{3}$ quienes encontraron una relación de H:M de 1,5:1 y con los de Momares B. y cols ${ }^{4}$ que revelaron una relación $\mathrm{H}: \mathrm{M}$ de 1,4:1. Estos hallazgos probablemente se deban a que los hombres presentan con mayor frecuencia el hábito de fumar y beber alcohol, los cuales son factores de riesgo para el cáncer oral.

La edad promedio de esta serie de casos se encuentra dentro del rango descrito por otros estudios, con una desviación estándar mayor a la evidenciada en otros artículos pudiendo ser secundario a características propias de la mues$\operatorname{tra}^{4,9-12,14,29-35}$. La edad promedio en hombres fue menor a la edad promedio en mujeres. Este hallazgo había sido observado por otros investigadores ${ }^{11,30,32,34}$. La mayor frecuencia de casos se encontró en el intervalo de pacientes mayores de 60 años (7/13) lo cual se corresponde con lo encon-

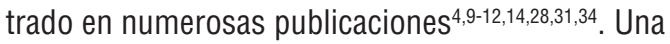
posible explicación para esto es que la exposición a los factores de riesgo aumenta con la edad 5 .

El consumo de tabaco se encontró en $25 \%$ (3/12) de los pacientes. Esta cifra es menor a la publicada por autores previos que reportan una frecuencia de hábito tabáquico en un rango de $37 \%$ a $85,3 \% 4,10,11,15,29,31-33$. Esta diferencia con lo observado en el presente estudio puede 
deberse a la falta de registro adecuado en las fichas clínicas utilizadas. El $100 \%$ de los pacientes fumadores correspondieron a hombres, lo cual pareciera ser consistente con investigaciones anteriores que describen una mayor frecuencia de hábito tabáquico en hombres en un rango de $73 \%$ a $98,2 \%$ 4,10,11,15,31,32.

Distintos autores mencionan que la presentación clínica más común es la úlcera, lo que concuerda con lo encontrado en esta serie de $\operatorname{cas}^{10,15,29,31,32}$.

El labio fue la localización más frecuente (4/13), seguido por la lengua (3/13). Estos hallazgos son similares a los encontrados por Guzmán P. y cols ${ }^{34}$ y por Arriagada 0. y cols ${ }^{14}$, quienes reportaron el labio como la localización más frecuente, seguido de la lengua. En contraste con los resultados previos, la mayoría de los estudios describe una frecuencia menor en labio, dando cuenta de $5 \%$ a $18,4 \%$ del total de $\operatorname{casos}^{4,10-12,28,31-33}$. Una posible explicación para esto es que algunos estudios sólo incluyen la mucosa labial como localización en labio obteniendo una menor frecuencia que aquellos que consideran la totalidad del mismo. En el presente estudio no se pudo discernir acerca de la zona afectada en el labio debido al escaso registro en las fichas clínicas.

La mayor frecuencia de tumores bien diferenciados en la muestra coincide con lo reportado por trabajos previos $\mathrm{s}^{4,10,30,31,33}$.

El tamaño promedio de las lesiones presenta similitudes a los hallazgos encontrados por Guzmán P. y cols $^{34}$. Ellos observaron un tamaño promedio de 28 milímetros ( $\mathrm{mm}$ ), siendo $3 \mathrm{~mm}$ mayor el promedio en mujeres. Igualmente revelaron que las lesiones en labio miden en promedio menos que las intraorales. La diferencia encontrada en el tamaño promedio entre ambos sexos podría explicarse en parte por el mayor tiempo de retraso en el diagnóstico en mujeres ${ }^{23}$.

En relación al estado del tumor primario y de los nodos regionales, se encontró que la mayoría de los pacientes fue diagnosticado en etapas pT1 o pT2 $(75 \%)$ y sin metástasis en nodos regionales $(66,6 \%)$. Esto se corresponde con lo publicado por otros autores $4,10-12,16$.

Este trabajo se enfrentó a una serie de limitaciones debido a su carácter retrospectivo y al número limitado de casos. Se evidenció al momento de la recolección de datos, una falta de registro adecuado perdiendo así información relevante para un análisis más detallado. No hubo calibración de los examinadores por lo que podrían existir sesgos. El estudio es susceptible a un sesgo de selección dado que los casos incluidos pueden ser pacientes que presentaron una mayor sobrevida o manifestaciones clínicas más claras por lo que acudieron a un centro de salud. Finalmente, en relación al estudio de la sobrevida, los pacientes se encontraban en distintos estadios de la enfermedad, lo que pudo provocar un sesgo en el estudio de la sobrevida global.

El presente estudio se realizó en una sola institución y con un número escaso de pacientes, por lo que su validez externa es limitada.

\section{CONCLUSIÓN}

El carcinoma espinocelular oral, en la población atendida en el CRS Cordillera Oriente, tiene una baja prevalencia, afectando con mayor frecuencia a hombres y a mayores de 60 años. El hábito tabáquico asociado a CEC se encuentra con mayor frecuencia en hombres. De los pacientes diagnosticados en este centro, la mayor parte tiene una presentación clínica de úlcera crónica, con un tamaño promedio de $30 \mathrm{~mm}$, un grado histológico bien diferenciado, un estado primario del tumor poco avanzado y nodos regionales no afectados. El sitio más frecuente es el labio.

La sobrevida de los pacientes atendidos en este centro es de $57,9 \%$ a los 5 años. El 23\% de Ios pacientes fallece debido a CEC. Los resultados sugieren que los pacientes de sexo femenino, menores de 45 años, fumadores, con presentación de masa exofítica, con un menor grado de diferenciación histológico, con un estado avanzada del tumor primario, con presencia de metástasis en nodos regionales y en un etapa TNM avanzada, tienden a presentar una sobrevida más baja, en este centro.

La sobrevida observada en la muestra así como en la literatura nacional e internacional pone de manifiesto el mal pronóstico de un paciente que es diagnosticado con CEC, así como la importancia de realizar un diagnóstico precoz y tratamiento oportuno. Es responsabilidad tanto de odontólogos como médicos generales, así como de especialis- 
tas, destinar parte del control rutinario para realizar un examen acusioso de la mucosa oral, con énfasis en la población de riesgo, e instruir a los pacientes de cómo realizar un propicio autoexamen y al reconocimiento de signos de alarma. Asimismo se deben crear programas de tamizaje de esta enfermedad 0 ampliar la cobertura de los preexistentes y desarrollar protocolos de manejo de esta patología que faciliten una adecuada derivación y tratamiento. La información presentada es de utilidad para la identificación de la población en riesgo de CEC oral, pudiendo ayudar en la producción de programas de screening.

Finalmente, se requieren más estudios de sobrevida general de pacientes con CEC en Chile, de mayor tamaño muestral y generar más investigaciones de calidad en cuanto a la presentación clínica y su rol en el pronóstico de esta patología.

Agradecimientos a Dr. Sebastián Godoy Sagredo por su gran colaboración en el trabajo.

\section{BIBLIOGRAFÍA}

1. Cardemil F. Epidemiología del carcinoma escamoso de cabeza y cuello. Rev Chil Cir 2014; 66(6): 614-20.

2. Riera P, Martínez R. Morbilidad y mortalidad por cáncer oral y faríngeo en Chile. Rev Méd Chile 2005; 133: 555-63.

3. Feller L, Lemmer J. Oral squamous cell carcinoma: epidemiology, clinical presentation and treatment. Journal of Cancer Therapy 2012; 3: 263-8.

4. Momares B, Contreras G, Martínez B, Ávalos N, Carmona $L$. Sobrevida en carcinoma espinocelular de mucosa oral: análisis de 161 pacientes. Rev Chilena de Cirugía 2014; 66(6): 568-76.

5. Bedi R, Butterworth M, Craig G, Langdon J, Lowndes $P$, Nolan A. Opportunistic oral cancer screening: a management strategy for dental practice. BDR 2000; occasional paper; 6.

6. Bolesina N, Femopase F, López de Blanc S, Morelatto R, Olmos M. Oral squamous cell carcinoma clinical aspects. Universidad Nacional de Córdoba, Argentina, 2012.

7. Johnson N, Franceshi S, Ferlay J, Ramadas K, Schmid S, MacDonald DG. Tumours of the oral cavity and oropharynx. World health organization classification of tumours. Lyon: IARC press, 2000; 163-75.

8. Sapp P, Eversole L, Wysockı G. Patología oral y maxilofacial contemporánea (2da edición). Editorial Elsevier, 2005.

9. Takahashi M, Aoki T, Nakamura N, Ogura G, KIKUCHI T, KIKUTI Y ET AL. Clinico pathological analysis of 502 patients with oral squamous cell carcinoma with special interest to distant metastasis. Tokai J Exp Clin Med 2014; 39(4): 178-85.

10. Silva L, Barbas do Amaral J, Vizcaíno J, Lopes C, OLIVEIRA F. A clinical-pathological and survival study of oral squamous cell carcinomas from a population of the north of Portugal. Med Oral Pathol Oral Cir Bucal 2014; 19(2): 20-6.

11. Arduino P, Carrozzo M, Chiecchio A, Broccoletti R, Tirone F, Borra E et al. Clinical and histopathologic independent prognostic factors in oral squamous cell carcinoma: a retrospective study of 334 cases. J Oral Maxillofac Surg 2008; 66: 1570-9.

12. GRImm M. Prognostic value of clinicopathological parameters and outcome in 484 patients with oral squamous cell carcinoma: microvascular invasion $\left(\mathrm{V}_{+}\right)$is an independent prognostic factor for OSCC. Clin Transl Oncol 2012; 14: 870-80.

13. Jan JC, Hsu W, Wong Y, Poon C, Jiang R, Jan JS ET AL. Prognostic factors in patiens with buccal squamous cell carcinoma: ten-year experience. J Oral Maxillofac Surg 2011; 69(2): 396-404.

14. Arriagada 0, Venegas B, Cantín M, Zavando D, Manterola C, Suazo I. Rol de las características clínicas e histológicas como factores pronósticos para la sobrevida en pacientes con carcinoma de células escamosas de la cavidad oral. Rev Chilena de Cirugía 2010; 62(5): 441-8.

15. Oliveira M, Wagner V, Filho M, Carrard V, Hugo F, Martins M. A 10-year analysis of the oral squamous cell carcinoma profile in patients from public health centers in Uruguay. Braz Oral Res 2015; 29(1): 1-8.

16. Wang $B$, Zhang $S$, Wang $X$. The recurrence and survival of oral squamous cell carcinoma: a report of 275 cases. Chin J Cancer2013; 32(11): 614-8.

17. Sklenicka S, Gardiner S, Dierks E, Potter B, Bell B. Survival analyses and risk factors for recurrence in oral squamous cell carcinoma: does surgical salvage affect outcome? J Oral Maxillofac Surg 2010; 68: 1270-5. 
18. Garzino-Demo P, Dell'acqua A, Dalmasso P, Fasolis M, La Terra Maggiore G, Ramieri G et al. Clinicopathological parameters and outcome of 245 patients operated for oral squamous cell carcinoma. Journal of Cranio-Maxillofacial Surgery 2006; 34: 344-50.

19. Rogers S, Brown J, Woolgar J, Lowe D, Magennis $P$ y ShaW R ET AL. Survival following primary surgery for oral cancer. Oral Oncology 2009; 45: 201-11.

20. Oliveira L, Ribeiro-Silva A, Oliveira J, Luiz A, Sala M, Zucoloto et al. Prognostic factors and survival analysis in a sample of oral squamous cell carcinoma patients. Oral Surg Oral Med Oral Pathol Oral Radiol Endod 2008; 106: 685-95.

21. Massano J, Regateiro F, Januário G y Ferreira A. Oral squamous cell carcinoma: review of prognostic and predictive factors. Oral Surg Oral Med Oral Pathol Oral Radiol Endod 2006; 102: 67-76.

22. Vallecillo-Capilla M, Romero-Olid MN, OlmedoGaya MV, Reyes-Botella C, Bustos-Ruíz V. Factors related to survival from oral cancer in an Andalusian population sample (Spain). Med Oral Patol Oral Cir Bucal 2007; 12(7): 518-23.

23. Yu T, Wood R, Tenenbaum $H$. Delays in diagnosis of head and neck cancers. Journal of the Canadian Dental Association 2008; 7(1): 1488-2159.

24. Dissanayaka W, Pitiyage G, Kumarasiri P, Liyanage R, Dias K, TIlakaratne W. Clinical and histopathologic parameters in survival of oral squamous cell carcinoma. Oral Surg Oral Med Oral Pathol Oral Radiol 2012; 113: 518-25.

25. Fernandes R, Barboza C, Clebis N, Moura S, Costa A. Prognostic significance of the anatomical location and TNM clinical classification in oral squamous cell carcinoma. Med Oral Patol Oral Cir Bucal 2008; 13(6): 344-7.

26. Shaw R, McGlashan G, Woolgar J, Lowe D, Brown J, Vaughan E, Rogers S. Prognostic importance of site in squamous cell carcinoma of the buccal mucosa. British Journal of Oral and Maxillofacial Surgery 2008; 47: 356-9.
27. Thomas B, Stedman M, Davies L. Grade as a prognostic factor in oral squamous cell carcinoma: A population - based analysis of the data. Laryngoscope 2014; 124: 688-94.

28. Weatherspoon D, Chattopadhyay A, Boroumand $S$, García I. Oral cavity and oropharyngeal cancer incidence trends and disparities in the United States: 2000-2010. Cancer Epidemiology 2015; 39: 497-504.

29. Kaing L, Manchella S, Love C, Nastri A, Wiesenfeld D. Referral patterns for oral squamous cell carcinoma in Australia: 20 years progress. Aust Dent J 2015; doi: 10.1111/adj.12314 .

30. Larsen S, Johansen J, Sorensen J, Krogdahl A. The prognostic significance of histological features in oral squamous cell carcinoma. J Oral Pathol Med 2009; 38: 657-62.

31. Álvarez E, Preciado A, Montoya S, Jiménez R, Posada A. Características clínico-histopatológicas del carcinoma escamocelular bucal, Colombia. Revista Cubana de Estomatología 2010; 47(1): 81-95.

32. Pires F, Ramos A, Oliveira J, Tavares A, Luz P, Santos T. Oral squamous cell carcinoma: clinicopathological features from 346 cases from a single oral pathology service during an 8 -year period. J Appli Oral Sci 2013; 21(5): 460-7.

33. Meza G, Muñoz J, Páez C, Cruz B, Aldape B. Carcinoma de células escamosas de la cavidad bucal en un centro de tercer nivel de atención social en la ciudad de México. Experiencia de cinco años. Av Odontoestomatol 2009; 25: 1928.

34. Guzmán P, Villaseca M, Antonio L, Araya J, Aravena P, Cravero C et al. Carcinoma epidermoide oral y orofaríngeo. Estudio clínico-patológico. Rev Chilena de Cirugía 2011; 63(3): 250-6.

35. Rivera C, González-Arriagada W, Loyola-Brambilla M, Paes de Almeida 0, Coletta R, Venegas B. Clinicopathoological and inmunohistochemical evaluation of oral and oropharyngeal squamous cell carcinoma in chilean population. Int J Clin Exp Pathol 2014; 7(9): 5968-77. 